\title{
Catástrofe, Subjetividad Femenina y Reconstrucción: Aportes y Desafíos desde un Enfoque de Género para la Intervención Psicosocial en Comunidades Afectadas por el Terremoto
}

\author{
Catastrophe Feminine Subjetivity and Reconstruction: Contributions and Challenges \\ From a Perspective of Gender for the Psychosocial Intervention in Communities Affected \\ by the Earthquake
}

\author{
Irene Magaña Frade* \\ Sebastián Silva-Nadales \\ Universidad de Santiago de Chile, USACH \\ Rayen Rovira Rubio. \\ Universidad Autónoma de Barcelona, España \\ (Rec: 5 de agosto de 2010 / Acep: 21 de octubre de 2010)
}

\begin{abstract}
Resumen
El presente artículo pretende aportar, desde un enfoque de género, al desarrollo de las intervenciones psicosociales y a la comprensión de la problemática que -en la subjetividad de las mujeres- suscita la catástrofe desencadenada por el terremoto y tsunami del 27 de Febrero del 2010. Desde una metodología cualitativa se realizó un análisis micro-social, cuyo dispositivo metodológico consideró: 1. Entrevistas en profundidad a mujeres dirigentas sociales de organizaciones de las regiones del Maule y Bío-Bío; 2. Revisión de datos de fuentes secundarias; 3 . Opinión de expertas en temas de género. Los resultados obtenidos son puestos en discusión con el modelo propuesto por Gaborit (2006) y dan cuenta de la conformación de una "Subjetividad Femenina Terremoteada" que, producto de la desarticulación de los espacios de cuidado, reconstruyen su subjetividad por medio de la participación comunitaria, volviéndose protagonistas en la reconstrucción de la vida cotidiana.
\end{abstract}

Palabras clave: Subjetividad femenina, tareas de cuidado, participación comunitaria, terremoto y reconstrucción.

\begin{abstract}
This paper intends contribute, from a gender approach, to the development of psychosocial interventions and understanding of the problematic-that the subjectivity of women- raises the disaster triggered by the earthquake and tsunami of February 27, 2010. From a qualitative methodology was carried out micro-social analysis, which methodological device considered: 1. In-depth interviews with women social leaders of organizations of the regions of Maule and Bio-Bio 2. Review of secondary sources, 3.Opinion of experts on gender issues. The results are placed in discussion with the model proposed by Gaborit (2006) and show the formation of a "female subjectivity's earthquake ", that product of the breakdown of care spaces, reconstruct their subjectivity through community participation, becoming protagonists in the reconstruction of everyday life. Key words: Female subjectivity, care tasks, community participation, earthquake and reconstruction.
\end{abstract}

Correspondencia a: Dra. Irene Magaña email: irene.magana@usach.cl 


\section{Introducción}

El 27 de febrero del 2010, a las 3:34 AM Chile sufrió el terremoto y tsunami más grande en la historia de la humanidad (magnitud 8,8 escala Richter), el que afectó al $80 \%$ de la población del país, dañando seis regiones, con epicentro en dos de las más pobres: Maule y Bío-Bío (MIDEPLAN, 2010). El desastre generado por el cataclismo destruyó estructuralmente ciudades y pueblos, desarticulando, tanto en lo público como en lo privado, los distintos sistemas sociales y vínculos cotidianos establecidos entre las personas y comunidades que convivían en los territorios afectados, generando rupturas en las estructuras de trabajo, en las familias y grandes cambios de relaciones dentro de las comunidades y la sociedad en general (Malcorra, 2010).

Los terremotos son imprevistos, repentinos, desatan en pocos segundos el desastre, y en este sentido podemos decir que poseen características particulares, que afectan psicológicamente más que otras catástrofes (McCaughey, Hoffman \& Llewellyn, 1994, citado en Gaborit, 2006). Su carácter impredecible genera un sentimiento de indefensión que aumenta la ansiedad y la incertidumbre cognitiva, que deja sin saber qué hacer y cómo protegerse cuando sucede el desastre. A ello se ha de agregar la existencia de réplicas, que crean condiciones que hacen revivir el trauma original, y que tienden a elevar aún más el estado de ansiedad de las víctimas, tal como también profundizan la incertidumbre y alerta, tienden a debilitar los roles sociales ejercidos cotidianamente por las personas, y además tienen el efecto de prolongar la posibilidad de asunción de roles reparatorios, como función de la prolongación del tiempo de restablecimiento de la normalidad, función decisiva también en la vivencia de daño que experimentará el individuo.

El carácter traumático de un desastre de esta naturaleza es innegable, y el impacto psicosocial que involucra permanecerá entre los afectados, especialmente cuando los recursos económicos y financieros de la nación que sufre esta catástrofe son insuficientes para hacer frente a las secuelas materiales y psicológicas del evento. Si bien la magnitud de este tipo de catástrofes no es posible de reducir a los números de las tragedias personales y a la destrucción material de viviendas e infraestructura pública, las diferencias entre países pobres y países ricos ilustran que los desastres no deben conceptualizarse exclusivamente como naturales. No es la fuerza de la naturaleza lo que explica tantas muertes y pérdidas, sino más bien las acciones de las personas, las políticas sociales del Estado y las responsabilidades privadas en la construcción de las viviendas, son las que hacen que las fuerzas de la naturaleza dejen o no tanta destrucción en su paso (Gaborit, 2006). Esto nos lleva a conceptualizar esta catástrofe como natural y social.

A su vez, resulta fundamental comprender el impacto psicológico que estos desastres generan, dado que entender el cómo y a quiénes afecta la catástrofe permite delinear las intervenciones psicosociales de manera oportuna, comprensiva, efectiva y adecuada a las necesidades sociales y subjetivas de los distintos grupos afectados -diferencialmente- por la catástrofe.

Desde ello, en el transcurso de los primeros meses post-terremoto las comunidades afectadas se enfrentaron al desafío de la reconstrucción de la vida cotidiana, motivo por el cual desde un primer momento se movilizó tanto el Estado como las comunidades, en torno al restablecimiento de servicios básicos, la entrega de alimentación, ropa y habilitación de albergues, escuelas, hospitales, guarderías y otros.

En relación a estas primeras reacciones de reconstrucción y reorganización de la vida cotidiana, es importante relevar el lugar que ocupan las mujeres y la densidad psicosocial de su accionar, ejerciendo un rol protagónico en las comunidades afectadas, para organizar a sus grupos familiares en función de la protección de los/as hijos/as, huir del peligro, buscar alimentos, ser soporte afectivo para otros y apoyar la organización requerida para la situación de catástrofe (Pezoa Navarro, 2010), siendo entonces las articuladoras fundamentales en las actividades comunitarias de reconstrucción, sosteniendo el clivaje de las iniciativas de apoyo y viabilizando la relación entre lo público y lo privado.

En este contexto, tanto la investigación como la intervención psicosocial se hacen fundamentales. La revisión de la literatura actual relacionada con el quehacer y el pensar psicológicos en situaciones de catástrofes revela un modo de hacer de la psicología que repite en su elaboración de modelos de intervención, la reproducción de una praxis efectista, que en su necesidad de soluciones inmediatas, constriñe las posibilidades comprensivas de la complejidad social y subjetiva que necesariamente están implícitas en este tipo de situaciones. Así, la literatura en la disciplina reporta intervenciones marcadas por la heterogeneidad y discontinuidad en sus aproximaciones teóricas y por el desarrollo de modelos que basan su legitimidad en resultados que -si bien son consistentes y apoyados en la evidencia- no necesariamente se encuentran vinculados a corpus teóricos que sustenten su capacidad heurística, ni su aplicabilidad para responder a la diversidad de expresiones y manifestaciones sociales y subjetivas que se producen en las personas afectadas por el terremoto; ni que ayuden a desarrollar enfoques comprensivos y de mejor empoderamiento de las comunidades locales para promover el afrontamiento integral de la situación desencadenada por la catástrofe.

A su vez, si realizamos una revisión de las experiencias reportadas para la intervención psicosocial en catástrofes, encontramos un interés focalizado en la definición de sectores vulnerables de la población afectada, caracterizando a niños/as, ancianos/as y adolescentes como los sectores priorizados para el cuidado, y a las mujeres, como vehículo para realizarlo. Así, resultan escasos e insuficientes tanto 
los reportes de investigación como de intervención que tengan como foco a las mujeres, si consideramos además, que en sectores populares y de pobreza ellas constituyen una población especialmente vulnerable.

Por otra parte y como los estudios sobre el género lo demuestran y describen, cuando se trata de mujeres, no solamente no son visibilizadas como vulnerables, sino que, además, son veladas por el papel de "cuidadoras" que la construcción social atribuye a lo femenino, imponiendo la carga de cumplir con la responsabilidad de ser el soporte material y psicológico de los otros, sumando así a su propia sensación de indefensión, el sentimiento de no poder cumplir con su rol de cuidado y protección respecto de los hijos y de la familia.

De esta manera, el presente artículo pretende aportar, desde un enfoque de género, al desarrollo de las intervenciones psicosociales y a la comprensión de la problemática que suscita la catástrofe desencadenada por el terremoto y tsunami del 27 de Febrero, relevando la preocupación por el lugar de anonimato e intermediación en que consideramos son ubicadas las mujeres, en la construcción e implementación de modelos psicosociales de intervención con que usualmente se responde, tanto desde las políticas públicas como desde disciplinas sociales como la Psicología. Así, en este artículo intentamos posicionar la particularidad con que es vivenciada por las mujeres su experiencia ante el terremoto y la posición que ocupan en la reconstrucción de la vida cotidiana, tanto en espacios familiares como socio-comunitarios.

\section{Antecedentes contextuales}

Desde que alrededor de los años sesenta surge en el campo de las ciencias sociales la teoría del género. Esta ha introducido un recorte analítico sobre la subjetividad femenina que ha permeado de manera transversal y distinta los últimos desarrollos disciplinares de la investigación social, desnaturalizando las diferencias entre hombres y mujeres y aportando a la visibilización de las relaciones culturales y de poder que allí se construyen. No obstante esta inclusión teórica, existen aún importantes brechas a la hora de sustentar modelos capaces de proporcionar lecturas comprensivas y orientaciones para las intervenciones psicosociales, que se encuentran indefectiblemente atravesadas por estas consideraciones teóricas, culturales y políticas.

Recientes son los intentos por incluir el género en materias de políticas sociales y públicas, con el fin de equiparar las posibilidades de acceso a recursos materiales y simbólicos, a la inclusión de la mujer en el espacio público y al reconocimiento de la diferencia; intentando así transversalizar el género más allá de las políticas específicas para las mujeres (CEPAL, 2010). Sin embargo, es posible constatar una deuda sustantiva, que hace aún importante seguir subrayando desde el género las diferencias, ausencias y velamientos con los que se construyen modelos psicosociales para la comprensión e intervención de la complejidad contextual implicada en fenómenos como los desastres naturales y terremotos.

Desde allí que se hace necesario pensar en las formas particulares y diversas con que las mujeres vivieron el terremoto y tsunami del pasado 27 de febrero del 2010 y la catástrofe socio-natural que conlleva la reconstrucción material y social de la vida cotidiana.

Cabe considerar que tradicionalmente la participación femenina ha ocurrido y ocurre restringiéndose fundamentalmente al ámbito privado de la producción y reproducción de la vida familiar, desarrollándose así, el área de ocupación de las mujeres alrededor del hogar: la producción de cuidados, la educación de los hijos, los temas de salud y bienestar social, la higiene y otros, situaciones preferentes de las mujeres producto de la diferenciación social del trabajo en el que han sido ubicadas, relegándose así al espacio doméstico (Aguirre, 2009).

En la particularidad del terremoto, es posible afirmar que este genera una situación de vulneración particular en las mujeres, vinculada a los roles de género, especialmente por el papel de cuidadora y protectora de la familia. La pérdida de las viviendas, de los medios de vida, la presión para encontrar comida y refugio, tienen un impacto en sus vidas y en desempeño roles dentro de la comunidad (Malcorra, 2010).

Así, en el caso de las acciones post-terremoto de las mujeres, el cuidado y las responsabilidades familiares continúan siendo actividades no reconocidas ni valoradas socialmente, pese a que estas son realizadas fuera del espacio doméstico y articulando protagónicamente al espacio comunitario en el que -esta vez por la situación de catástrofe - se sustenta la vida cotidiana. Pese a la forzada salida del hogar, se mantiene invisibilizadas a las mujeres y su capacidad de agencia y actoría ciudadana, que constituye una base invisible para el bienestar social y que en distintas oportunidades no es considerada suficientemente como una dimensión necesaria de abordar desde las políticas públicas (Aguirre, 2009). No obstante el protagonismo de las mujeres es fundamental -desarrollando múltiples acciones locales desde la autogestión y respondiendo a los incentivos otorgados desde el Estado por medio de programas como el Plan Nacional de Reconstrucción "Mujer levantemos Chile"estas son ubicadas como mediadoras en la reconstrucción; entre el Estado, los gobiernos locales y las comunidades y las familias (Sapag, 2010).

Desde la intervención psicosocial y en cuanto al trauma vinculado a una catástrofe de esta envergadura, comprendemos que los traumas se generan a partir de eventos peligrosos y repentinos que sobrepasan los recursos psicológicos, físicos y económicos de las personas y las comunidades, siendo de gran intensidad, impredecibles, infrecuentes 
y variantes en duración, de agudos a crónicos, pudiendo afectar a una persona o a una comunidad (Gaborit, 2006).

Las reacciones comunes que se presentan posteriormente a un hecho traumático se pueden dividir: en aquellas que aparecen en el periodo de emergencia inmediata; las que exhiben las personas después de 72 horas, hasta pocas semanas del evento traumático; y las secuelas a largo plazo. Desde ello hay respuestas que se encuentran en los límites de la normalidad considerando las circunstancias y siendo una dificultad el hecho de que las reacciones perduren por un tiempo más allá del momento en que son útiles y funcionales. En ese caso resultan una limitación para que la persona asuma su vida con normalidad, perturbando las relaciones interpersonales y la capacidad de manejar las dificultades no asociadas con el evento traumático, es en esas circunstancias que se puede hablar de estrés post traumático (García \& Mardones, 2010).

De acuerdo a lo anterior y siguiendo a Gaborit (2006), se propone un modelo explicativo de las reacciones traumáticas ante el desastre, donde se asume que si bien los seres humanos no respondemos del mismo modo a las situaciones de catástrofes, hay una serie de factores identificables que determinan las distintas posibilidades de reacción. Así, se propone un modelo que ayuda a explicar cómo reacciona una persona ante un desastre, los factores que potencian o mitigan sus efectos, y aquellos otros que ayudan o entorpecen la recuperación psicosocial del individuo. En este modelo resulta fundamental el considerar cómo la clase del trauma y su severidad incide en cómo el individuo genera sus respuestas, dado que la severidad del trauma determina la profundidad del mismo y el tiempo y curso de su recuperación. Posteriormente se identifican lo que se denominan mediadores sociales, entre los cuales tres resultan fundamentales: el apoyo social para afrontar el trauma, donde mientras mayor sea la red percibida mejor y más rápida la recuperación, en relación a aquellas que se encuentran aisladas socialmente; las estrategias de afrontamiento, que se comprenden como las acciones dirigidas a la solución, centradas en el impacto emotivo causado por el acontecimiento traumático. Finalmente, se identifica como fundamental el significado con que las personas revisten el acontecimiento.

Considerando que este modelo otorga un lugar central a los significados subjetivos con que las personas vivencian la catástrofe, permite un nivel de flexibilidad que posibilita la inclusión de consideraciones de género, necesarias para una adecuada lectura del efecto del terremoto y tsunami en las mujeres de las regiones del Maule y Bío-Bío y además orientar las intervenciones que se realicen con ellas.

\section{Método}

En este estudio se utiliza, desde una perspectiva comprensiva, una metodología cualitativa, planteándose desde una visión hermenéutica e interpretativa un marco de referencia que permita buscar significados y percibir la realidad de las personas y sus contextos como un todo (Ruiz Olabuenaga, 1996). Busca, así, propiciar una mirada reflexiva y propositiva acerca de las problemáticas sociales suscitadas por el terremoto, resaltando la necesidad de marcos interpretativos como el proporcionado por el enfoque de género, de manera de tener mayores herramientas para la interpretación de la realidad.

El diseño de la investigación que intenciona el carácter exploratorio de un análisis microsocial, enfatiza la comprensión de las relaciones y vínculos de las personas con su entorno físico y social. Considera sus acciones y desempeños desde sus posiciones o inserciones sociales, así como sus valores y creencias hacia el medio y hacia sí mismo, y sus interpretaciones de la experiencia cotidiana, privilegiando el estudio de la agencia humana (Freidin, 2004).

El dispositivo metodológico considera tres etapas: 1.Detección de sujetos pertinentes y realización de entrevistas para la obtención de información, entre mujeres participantes en organizaciones sociales de las zonas afectas por el terremoto (Regiones del Maule y Bío-Bío). 2.- Revisión de documentación y estudios realizados acerca de experiencias de mujeres afectadas por el terremoto, utilizando como fuente de datos dos observatorios (El Observatorio Género y Equidad (UNDEF y UNIFEM), y el Observatorio de Género de la CEPAL) 3.- Sistematización, triangulación y análisis de toda la información recopilada, con el respaldo de la opinión de dos expertas en el tema (obtenido a través de entrevistas en profundidad).

En la primera fase de la investigación, se seleccionaron las informantes claves mediante un muestreo guiado teóricamente, cuyos criterios principales fueron: ser mujeres adultas, haber sido damnificadas por el terremoto, ser habitantes de las regiones del Maule o Bío-Bío y participar de organizaciones sociales comunitarias y/o de mujeres. Siguiendo estos criterios se realizaron siete entrevistas en profundidad, que fueron sometidas a un análisis categorial asistido informáticamente por el ATLAS ti, versión 6.1.

Luego, en base a los resultados del análisis de las entrevistas y al modelo propuesto por Gaborit (2006) se construyó una guía de registro que propone dos ejes de análisis: Los efectos subjetivos del terremoto y la participación comunitaria como mediador social (que a su vez contempla: el significado, el apoyo social y las estrategias de afrontamiento), con los cuales se integraron las categorías para el análisis del material secundario. Este material secundario de análisis provino de dos fuentes: el Observatorio Género y Equidad (UNDEF y UNIFEM) y el Observatorio de Género de la CEPAL, el que fue analizado en base a estas guías de registro propuestas para el efecto, permitiendo contrastar dicho material y triangularlo con las distintas fuentes de información.

Finalmente, y a modo de enriquecer la reflexión sobre la pertinencia del enfoque de género para el análisis de la 
condición de las mujeres afectadas por el terremoto y el diseño de intervenciones psicosociales apropiadas para el caso, se triangularon los resultados previamente obtenidos, con la opinión de expertas en la materia, ejercicio con el cual se afianzaron las dos dimensiones de análisis: subjetiva y participativa. Estas dimensiones representan los ejes de reflexión fundamental en torno a los cuales analizamos la problemática del género en el contexto de catástrofe, sustentando, de este modo, un proceso de reflexividad transversal que estará implícito en todas las discusiones del estudio.

\section{Resultados}

El modelo que se presenta a continuación condensa los resultados emergentes del proceso de investigación en sus tres fases, organizados de manera de aportar a la comprensión de los efectos subjetivos de las mujeres en la catástrofe suscitada por el terremoto y tsunami. A la vez se consideraron aspectos del Modelo de Gaborit (2006), poniéndolos en diálogo con nuestros resultados.

Como Subjetividad Femenina Terremoteada hemos designado al impacto subjetivo en las mujeres afectadas por el terremoto y tsunami del 27 de febrero del 2010. La forma en que se manifiestan estos cambios subjetivos son muy variados, pero es posible extraer algunas caracteristicas comunes, tanto de los elementos emergentes de las entrevistas como de lo señalado por las expertas en la materia y en los observatorios de género.

Primeramente, resulta importante considerar que el espacio del hogar ha constituido tradicionalmente, el lugar en que se desarrollan gran parte de las labores de las mujeres, incluso en aquellos casos en que estas trabajan remuneradamente fuera de él y/o están insertas en el espacio público debido a su participación comunitaria. Así, la vivencia de destrucción o daño de sus viviendas es significada como "lo perdimos todo", lo que marca un fuerte sentimiento de pérdida que no es posible de entender tan sólo como una pérdida material, sino como la destrucción o "resquebrajamiento" de los elementos que constituyen su subjetividad e identidad, dada la centralidad del "ser madres" y el ejercicio de los cuidados en su constitución subjetiva.

Sin embargo, en muchos casos esta sensación de pérdida total, vinculada al desplazamiento de los ejercicios de cuidado hacia el espacio comunitario, estaría relacionado a las Condiciones Pre-Terremoto. Es decir, en la medida en que las mujeres tienen algún nivel de participación comunitaria, estas experiencias se vuelven centrales para la reorganización de la vida cotidiana que en muchos casos se volcó hacia el espacio comunitario. De esta manera, la participación, el apoyo mutuo y la preocupación-ocupación de los cuidados del otro se vuelven el sustento subjetivo de las mujeres, y funcionan como factor protector en dos sentidos: de sí mismas y de otros/as.

Figura 1: Subjetividad Femenina Terremoteada

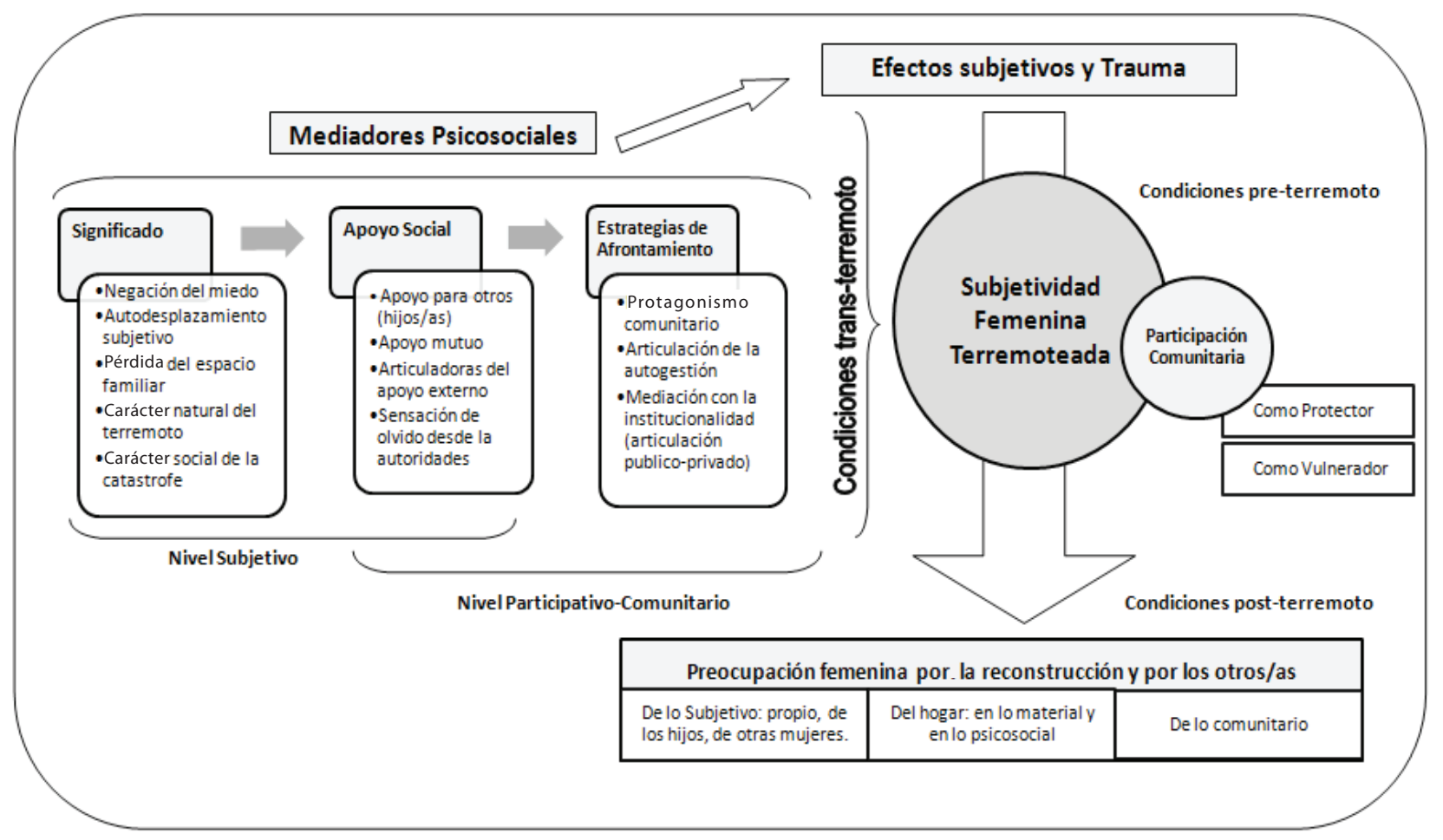


Pese a esta condición protectora de la participación, es posible advertir que en muchos casos esta puede generar niveles de vulneración para quienes las sustentan, a propósito del desgaste físico y psicológico que implica la organización, articulación y ejecución de las tareas antes desarrolladas en el espacio doméstico y desplazadas hacia lo comunitario, tomando en consideración las condiciones precarias y la dificultad que implica un estado de ansiedad generalizado, que hace difícil la organización de las tareas, la distribución la ayuda y la consiguiente satisfacción de las necesidades básicas. Esta situación acrecienta su condición de vulneración, cuando se vuelven tareas exclusivas del quehacer femenino, y que a su vez se cronifican cuando son ubicadas como mediadoras y organizadoras del apoyo provenientes de otros sectores del país. Este doble anclaje subjetivo de la participación como factor protector y de vulneración constituye un elemento central de las Condiciones Post-Terremoto, para la subjetividad de las mujeres.

En el modelo propuesto se consideraron tres mediadores psico-sociales que gravitarán sobre los efectos psicológicos de la situación de desastre, y que sirven para entender el desarrollo de la Subjetividad Femenina Terremoteada.

El primer mediador psicosocial, que hemos ubicado en el nivel subjetivo de la experiencia es El Significado atribuido al terremoto y tsunami, desde donde las mujeres en cuestión ponen el acento en el carácter natural e impredecible de la catástrofe, sin embargo, enfatizan las consecuencias sociales que este fenómeno tiene. Es decir, hay una claridad acerca de que el efecto devastador de este, tiene que ver con las condiciones pre-terremoto, asociadas a las circunstancias de vida de los sectores más pobres que fueron afectados por la catástrofe. De esta manera, las mujeres entrevistadas no comprenden la devastación del terremoto y tsunami como mera consecuencia de "la fuerza de la naturaleza", sino que también la visualizan como producto de las condiciones de habitabilidad de sus viviendas, especialmente por los usos del suelo y la calidad de las construcciones.

También en relación a la condición social de la catástrofe y sus efectos, las mujeres entrevistadas muestran preocupación especial por el desempleo, que las afecta tanto a ellas como a los hombres. A su vez, lo relacionan el aumento del alcoholismo como consumo masculino, situación que haría aumentar la violencia intrafamiliar.

"Para mi gusto, los principales problemas que vamos a vivir son la falta de trabajo. Primero pierden el trabajo, se ponen a tomar y ligerito llega la violencia."

Por otra parte, señalan cómo tuvieron que desplazarse a sí mismas y las posibilidades de expresar y elaborar sus sentimientos, para seguir siendo el sostén de sus familias y ejercer las tareas de cuidados.

"no teníamos ni tiempo para sentir miedo o descansar, si no eran nuestros hijos, eran los de otras, ver cómo pararíamos la olla entre todos o como repartiríamos la ayuda...”.

"Los derechos de las mujeres los veo en riesgo. Al ser las sustentadoras del hogar, sentimos que perdemos nuestros derechos, porque no tienes derecho ni a llorar. No hay tiempo para decir: pucha qué me está pasando. Debemos ser las fuertes de la casa y con eso ya has perdido tu derecho de sentir, de llorar, que es tan natural en el ser humano".

En relación al Apoyo Social, la dificultad se presenta en la medida en que son ellas -en su condición de dirigentasquienes vehiculizan el apoyo social, lo que nuevamente funciona de manera paradojal: tanto como factor protector, como factor de vulneración.

La condición de vulneración estaría dada porel desplazamiento de las propias necesidades, en primer lugar hacia espacio privado-familiar, en que quedan ubicadas como las articuladoras del bienestar psicosocial de la familia y de los ejercicios de cuidado. Y en segundo lugar hacia el espacio comunitario, donde producto de la destrucción material del espacio doméstico, se desarrollan y cubren las necesidades básicas de abrigo, alimentación y contención psicosocial.

"Ellas fueron el soporte de todo, pero a ellas nadie las sostuvo, es decir, su propia fuerza de mujer es la que las hacía caminar y lograr lo que se ha hecho hasta el momento"

Así, las dirigentas sociales advierten sobre de las dificultades con que las mujeres vivencian la catástrofe, volviéndose el apoyo para sus pares de género, lo que denominamos Apoyo Mutuo y que se da en distintos niveles: en la propia familia, en la comunidad y hacia otras mujeres.

"Muchas andaban como si trajeran un saco en la espalda: no se peinaban, no les daban ganas de comer, nada, como si el mundo-su mundo-con el terremoto se hubiera acabado. Pero no es así. Nosotras les decimos: tú vales, tienes que salir adelante, quererte tú primero, y después ves a tu familia"

Tal como señalamos cuando hicimos referencia a la participación, son ellas en su condición de mujeres y de dirigentas quienes articularon el apoyo social por parte de la autoridades e instituciones locales y nacionales, lo que además del desgaste que esto produjo y produce en ellas, ha generado una fuerte sensación de desplazamiento y olvido, relacionada con las dificultades para repartir la ayuda de abrigo y comida, la mala calidad de las soluciones habitacionales temporales -que muchas creen que se han vuelto definitivas-, el escaso o nulo apoyo psicosocial y el olvido mediático del tema.

"Creo que el terremoto y todo lo que ha pasado después, a nosotras nos empobrece. Nos empobrece como sociedad, pero en especial a las mujeres. Es como que quieres ir adelante y no puedes. Debes dar un paso atrás, es nadar contra la corriente" 
El último elemento de lo que hemos denominado los Mediadores Psicosociales, corresponde a las Estrategias de Afrontamiento, las que por ser entendidas en el nivel participativo-comunitario, son posibles de remitir a elementos mencionados a propósito del valor de la participación y que podemos resumir en la siguientes ideas: El protagonismo femenino en el espacio comunitario, para la reorganización de la vida cotidiana y la reconstrucción; la articulación de la autogestión y el apoyo mutuo; y la mediación con las autoridades e instituciones, en la que quedan ubicadas como las articuladoras entre el espacio público y el privado (en muchos casos ampliado más allá del hogar, hacia la comunidad).

"Comenzamos a formar redes inmediatamente. Volvimos al trueque: tengo harina, tú azúcar, yo tengo fideos. No sólo hicimos ollas comunes. También compartieron pañales, ropa, toallas higiénicas y otros enseres fundamentales en la primera etapa de emergencia. Eso fue algo tan humano, no importa si te conocían o no."

Finalmente para entender los efectos subjetivos del terremoto y lo que hemos denominado la Subjetividad Femenina Terremoteada, resultan importantes las proyecciones de las mujeres en relación al proceso de reconstrucción, lo que emergió de manera espontánea en todas las entrevistas y que se condice con lo que advierten las expertas en género y el material de los observatorios consultados. Ellas se sienten y quieren seguir siendo partícipes activas del proceso de reconstrucción, enfatizando la preocupación por los otros y por los aspectos psicosociales de la reparación. A su vez, destacan el valor del apoyo mutuo y la participación comunitaria como ejes fundamentales para la reconstrucción de la vida cotidiana.

De esta manera, su preocupación por los otros en la reconstrucción y las necesidades psicosociales alcanza distintos niveles: el propio y el de las otras mujeres más afectadas por la situación; el de los hijos/as; el de los cónyuges (especialmente en relación a la generación de empleos); el de la reconstrucción del espacio familiar del hogar, tanto en lo material como en lo relacional. Considerando el valor de lo comunitario y la participación, como una experiencia de aprendizaje, a propósito de cómo hacer frente a situaciones de catástrofe de manera colectiva, posibilita un mayor bienestar psicosocial para los sujetos individuales y para las comunidades.

"Debemos unirnos e ir a pelear al municipio. Decir cuáles son nuestras necesidades, hacer cabildos y reuniones, asambleas, juntarnos, hacer sindicatos de mujeres donde podamos defendernos todas. Que nos reunamos, nos afiatemos y luchemos por nuestros derechos".

"Imagino que las mujeres tenemos harto que hacer en el proceso de reconstrucción. Pero también necesitamos amor, comprensión y cariño. Eso es fundamental, agregar a las casas que-esperamos- construir pronto, fuerza, dinamismo y esperanza."

\begin{abstract}
"Me gustaría que nos uniéramos más, que tuviéramos más capacitación e iniciativas para conocernos y apoyarnos. Que viéramos lo mejor de la otra persona y no lo peor".
\end{abstract}

\section{Discusión}

Con el fin de otorgar un valor interpretativo a los resultados anteriormente expuestos, volveremos sobre algunos de los hallazgos centrales del estudio, esta vez para vincularlos con las teorías de género y delinear sus alcances para el desarrollo -no solo de propuestas comprensivas- sino también para su aplicabilidad en el diseño e implementación de intervenciones psicosociales. A su vez, realizar algunas consideraciones para las políticas públicas orientadas a la reconstrucción, no sólo material, de la catástrofe suscitada por el terremoto, sino también en sus aspectos subjetivos y comunitarios, con una especial consideración por el lugar de las mujeres en este importante desafío.

En principio y tal como señala la literatura asociada al tema: mientras mayor es el apoyo social percibido por parte de los sujetos en situaciones de catástrofe, mayores son las expectativas subjetivas de recuperación del trauma. Inclusive, la percepción de un adecuado apoyo social permitiría la prevención de la instalación misma de dicho trauma.

Sin embargo, en el caso de las mujeres entrevistadas, encontramos una primera característica referida a la dificultad con que es percibido el apoyo social, puesto que en su condición de mujeres y de dirigentas sociales son ellas mismas quienes vehiculizan este apoyo social, lo que funciona subjetivamente de manera paradojal: como factor protector y como condición de vulneración.

Esta paradoja se desata, en primer lugar, porque implica un desplazamiento de las propias necesidades, con un doble anclaje: el primero dado en relación al espacio privadofamiliar, donde ellas quedan ubicadas en el lugar de articuladoras del bienestar psicosocial de la familia, sobre todo de los hijos y en muchos casos de sus cónyuges o parejas. El segundo anclaje dice relación al espacio comunitario, en el cual las responsabilidades y tareas previas a la catástrofe se acrecientan, a propósito de la destrucción material del espacio doméstico, volviéndose el espacio comunitario el lugar donde se desarrollan y cubren todas las necesidades básicas de abrigo, alimentación y contención psicosocial.

También en referencia al apoyo social, y en relación a la acción participativa, se produce una dilatación de las labores comunitarias, ya que las mujeres que contaban con experiencias de participación pre-catástrofe multiplican exponencialmente sus labores comunitarias a fin de hacer frente a la situación. Esto conlleva las connotaciones subjetivantes habituales para las mujeres, es decir, el hacerse cargo de los cuidados y necesidades de los otros, y participar en las tareas comunitarias incluso en contextos de gran 
conmoción y ansiedad social, sobre todo en los primeros momentos post-terremoto y tsunami.

Por otra parte, esta paradoja tiene la particularidad de atravesar tanto el eje subjetivo como el de la acción participativa, y afectar a las mujeres de manera generalizada -abarcando varios ámbitos de lo femenino- y también permanente -constituyendo una expresión de trauma-que repercute en las posibilidades de acción. Así, se produce una desestructuración de la subjetividad en tanto se perturba la posibilidad de ejercicio del rol de cuidadoras.

Pese a este efecto traumático que implica la pérdida material y subjetiva del espacio del hogar, las mujeres afectadas por la catástrofe mantienen una preocupación constante por el otro y en el caso particular de las dirigentas se ubican en el lugar de "madres o cuidadoras de las madres", mostrando, así, el peso que para las mujeres -incluso fuera del ámbito privado- tiene el ejercicio del cuidado. Aparece entonces, como articulador de la subjetividad femenina, la posibilidad de inclusión en el ejercicio del apoyo social y también la posibilidad de afrontamiento de la situación de trauma para las mujeres.

A su vez, es importante subrayar la especial condición de devastación psicológica que implica esta imposibilidad del ejercicio del cuidado para las mujeres, que además de su papel tradicional de cuidadoras de la familia ejercen la jefatura del hogar. En Chile existe un porcentaje importante de hogares que son liderados por mujeres, los que según la encuesta CASEN 2009 (MIDEPLAN, 2010) ascienden a un $31,5 \%$, situación que se acrecienta en condiciones de pobreza (según la misma encuesta 2009, el 47,9\% de los hogares indigentes y un $43,2 \%$ de los hogares pobres tienen a mujeres en su jefatura). Por tanto, son las mujeres jefas de hogares y pobres las particularmente afectadas por el terremoto, dado el papel central que desempeñan en términos de proporcionar cuidado y medios de subsistencia y mantenimiento de las estructuras familiares. En estos casos, el trauma provocado por la catástrofe vulnera no sólo su papel de cuidadoras y proveedoras, sino también su posición en términos de dignidad e integralidad como jefas de hogar, en tanto pierden la seguridad y estabilidad adquiridas en los contextos previos al terremoto.

Esta consideración hace especialmente necesario situar la perspectiva de género en la discusión sobre la intervención psicológica y social.

A fin de entender las dinámicas y funciones que las mujeres desarrollan como primeras estrategias de afrontamiento en la situación de post-terremoto, es necesario recordar la urgencia que impone la destrucción de la estructura física que constituye el "espacio propio" de la mujer, es decir el hogar, destrucción que implica una profunda alteración de las interacciones cotidianas y de las relaciones de poder que se han establecido tanto en el espacio interno, privado, como en el entorno directo en que se enclava la vivienda, alterando las relaciones entre los diferentes actores comunitarios.
Es necesaria la reconstrucción del espacio privado/ público, pero también -y con una importante gravitación para las mujeres- es importante restablecer las relaciones entre los individuos que lo habitan, ya que sin este soporte vincular y social, se pierde el sentido de la reconstrucción para el funcionamiento de las familias. Así, son las mujeres las que se encargan desde sus inicios de la difícil tarea de velar por el cuidado familiar; en un sentido ampliado resultan fundamentales en la reestructuración de las redes comunitarias y desde ahí en la satisfacción de las necesidades básicas de alimentación, salud y otros cuidados.

Estas acciones de afrontamiento, por su característica, están teñidas de significación subjetiva y precisamente desde ahí se articulan como modo inicial de soportar y enfrentar la situación traumática. En efecto, el posicionamiento de activo en la participación comunitaria, es lo que permite -una vez más- sostener el papel fundamental de cuidadoras que tradicionalmente ha dado sentido a la acción femenina y que permite además articular desde lo comunitario el espacio privado. Es entonces en los campos de la reconstrucción comunitaria donde se ejerce -en la catástrofe- la responsabilidad de la jefatura de hogar y el consiguiente cuidado de la estabilidad familiar.

Los datos obtenidos, tanto directamente de las mujeres entrevistadas como los de fuentes secundarias, nos permiten postular el rol central que la mujer juega en la reconstrucción comunitaria y la importancia que para la familia este rol tiene.

De otra parte, habría que advertir que nuevamente es el papel protagónico en la acción comunitaria el que deja a las mujeres atrapadas en una paradoja, pues a la vez que estas acciones las sostienen en lo subjetivo, las anulan allí donde su papel activo en el cuidado y sostén de los otros, las desplaza como sujetos reduciendo su valor a la utilidad como mediadoras de los cuidados dirigidos hacia la familia, principalmente a los/as hijos/as (niños y jóvenes). Esta utilidad mediadora de las mujeres permite visibilizar las inequidades de género presentes en la comunidad, y la desigualdad con que se distribuyen las responsabilidades entre hombres y mujeres, reificando acciones de exclusividad femenina, que serían muy difíciles de canalizar si no es a través de la acción de las organizaciones comunitarias sustentadas por la participación femenina.

Hay que destacar en ésta paradoja el papel desempeñado por el Estado en sus intervenciones hacia las mujeres, dada la direccionalidad e injerencia en reproducir diferencias de posiciones sociales entre hombres y mujeres.

Desde estas apreciaciones nuevamente se hace necesario introducir a la discusión de la intervención psicosocial, un enfoque de género, que por una parte permita visibilizar las diferencias hombres/mujeres y sus particularidades, y por otra, que no implique la cristalización y naturalización de los roles y tareas tradicionalmente atribuidas a hombres y mujeres, que hacen del cuidado, de lo privado y de los otros, 
tareas de exclusividad femenina. Habría que resaltar, además, las posibilidades que ofrece la situación post-terremoto y su subsecuente reconstrucción de vínculos comunitarios, en la posibilidad de subvertir las relaciones de inequidad de género con que se insertaban las mujeres en la comunidad, en la situación pre-terremoto.

En tanto el plano de los significados se encuentra siempre articulado tanto en el eje subjetivo como en el de la participación comunitaria, sólo es necesario recalcar algunos de los significados que funcionan como organizadores/desorganizadores de la subjetividad femenina en la situación de catástrofe.

Todos los datos apuntan a situar como central en la articulación de la subjetividad femenina terremoteada el quiebre que produce la imposibilidad del ejercicio del cuidado, evento que es vivenciado por las mujeres como traumatizante. En la medida que invade todas las instancias estructurantes de la condición materna y familiar desde la que se posicionan las mujeres, fractura la posibilidad de protección de los hijos y dificulta el hacerse cargo de la estabilidad familiar, se encuentra en este sentido "terremoteada".

Esta situación, es desestructurante de la integración identitaria y es significada como amenazante para el ejercicio materno, activando desde lo subjetivo una sobredemanda de control del cuidado, y a la vez, generando una sensación de incapacidad de ejercer de manera eficiente los lugares comunes de la construcción identitaria, quedando así atrapadas entre la visualización del riesgo objetivo que implica el terremoto y la imposibilidad subjetiva de responder desde lo esperado en el ejercicio materno.

Las mujeres, frente a esta vivencia de indefensión y fragilidad en la posibilidad de cuidado familiar y materno, han resignificado otros espacios para el ejercicio de estos roles. Es así como desde el análisis realizado, podemos inferir que para ellas se vuelven visibles espacios como los comunitarios para asumir los temas sentidos como propios: la reconstrucción de su casa, el cuidado de sus hijos, la alimentación de su familia y otros. Es decir, desde la situación de catástrofe, se significan nuevos "temas de mujer", apareciendo éstos como ampliación del espacio de lo femenino hacia responsabilidades compartidas.
Por otra parte, las mujeres cambian sus significaciones personales y se empoderan, en la medida que se hacen cargo de la reconstrucción del daño dejado por el terremoto, en estos nuevos espacios comunitarios. Así, pareciera que en situaciones de crisis, las mujeres pueden mostrar una capacidad extraordinaria para suplir las habituales seguridades establecidas a través de distintas iniciativas de subsistencia. Sin embargo, por muy eficaces y oportunas que estas iniciativas femeninas sean para la solución de respuestas a situaciones urgentes de la familia o comunidad, nada garantiza que en ese proceso se genere una opinión pública capaz de influir en la agenda política nacional, ni en la percepción social, que puedan subvertir la posición de vulnerabilidad de las mujeres.

\section{Referencias}

Aguirre, R. (2009). La Bases Invisibles del Bienestar Social. EL trabajo no remunerado e Uruguay. Montevideo: UNIFEM.

Carvajal, C. (2002). Trastorno por estrés postraumático: Aspectos clínicos. Revista Chilena de Neuro-Psiquiatría, 40, 22-34.

CEPAL (2010). ¿Qué género para que igualdad?: XI Conferencia Regional sobre la Mujer de America Latina y el Caribe. Brasilia, Julio 2010.

Freidin, B. (2004). El uso del enfoque biográfico para el estudio de las experiencias migratorias femeninas. En R. Sautu. (edt) El método biográfico. La reconstrucción de la sociedad a partir del testimonio de los actores. Buenos Aires: Lumiere.

García, F., \& Mardones, R. (2010). Prevención de trastorno de estrés postraumático en supervivientes del terremoto de Chile de febrero de 2010 : Una propuesta de intervención narrativa. Terapia Psicológica, 28, 85-93.

Gaborit, M. (2006). Desastres y trauma psicológico. Pensamiento Psicológico, 2 , 15-39.

Malcorra, S. (2010). What is the role of the United Nations, particularly for peacekeeping forces and missions in post-disaster situations? An overview from a gender perspective. En (re)construir la igualdad. Presented at the XI Conferencia Regional Sobre la Mujer de America Latina y el Caribe., Brasilia.

MIDEPLAN. (2010). Encuesta Casen 2009. La Encuesta de Caracterización Socioeconómica Nacional. Recuperado en Octubre 4, 2010 disponible en http://www.mideplan.cl/casen2009/

Pezoa Navarro, P. (2010). Mujeres: Ojo con los mensajes de reconstrucción. Recuperado en Octubre 4, 2010 disponible en http://www. observatoriogeneroyliderazgo.cl/index.php?option=com_content\&tas $\mathrm{k}=\mathrm{view} \& \mathrm{id}=2697 \&$ Itemid=2

Ruiz Olabuenaga, J. (1996). Metodología de la Investigación Cualitativa. Bilbao: Universidad de Deusto.

Sapag, B. (2010). Plan Nacional de Reconstrucción "Mujer Levantemos Chile". En reconstruir la igualdad. Presented at the XI Conferencia Regional Sobre la Mujer de America Latina y el Caribe., Brasilia. 
